\title{
Silicon and Sulphur Chemistry in the Inner Envelopes of Carbon Stars
}

\section{KAREN WILLACY and ISABELLE CHERCHNEFF}

Physics Department, UMIST, Manchester, U.K.

The chemistry of silicon-, sulphur-, and oxygen-bearing molecules is investigated in the inner envelope of a typical carbon-rich AGB star. The effect of pulsation-driven shocks on the gas close to the stellar photosphere is considered. The chemistry is governed by bimolecular and termolecular reactions between neutrals. Thermal equilibrium calculations predict small amounts of $\mathrm{SiS}$ and $\mathrm{SiO}$ molecules in stellar photospheres for $\mathrm{C} / \mathrm{O}$ ratios characteristic of carbon stars. On the other hand, radio maps show that these molecules are present with large abundances close to the star. Our model predicts the formation of $\mathrm{SiS}$ and $\mathrm{SiO}$ in the periodically shocked inner regions and shows that the inner envelope of an AGB star is a region of very active molecule formation. 